\title{
Intentional Fracture of Previously Placed Stents: Impact of Pre-stenting in a Piglet Model
}

\author{
András Bratincsák, MD, PhD ${ }^{1}$, William Van Alstine, DVM, PhD, DACVP³, Lindsay Koren, $\mathrm{BE}^{2}$, \\ Kimberly Stoughton, $\mathrm{CVT}^{3}$, José Negrón-Garcia, $\mathrm{CVT}^{3}$, Anthony Ragheb, $\mathrm{PhD}^{2}$, \\ Hannah El-Sabrout, HSDG ${ }^{4}$, John W. Moore, MD, MPH ${ }^{5}$, Howaida el-Said, MD, PhD ${ }^{5 *}$ \\ ${ }^{1}$ Kapi'olani Medical Center for Women and Children, University of Hawaii, Honolulu, Hawaii, United States \\ ${ }^{2}$ Cook Medical, Bloomington, Indiana, United States \\ ${ }^{3}$ Cook Research Incorporated, West Lafayette, Indiana, United States \\ ${ }^{4}$ Department of Molecular, Cell and Developmental Biology, University of California, Los Angeles, California, United States \\ ${ }^{5}$ Rady Children's Hospital, University of California San Diego, San Diego, California, United States
}

\begin{abstract}
Background: Intentional stent fracture in vivo induces medial dissection/vessel injury. Spontaneous stent fracture in humans can lead to stent collapse, hemodynamic compromise, and embolization of stent fragments, which could be prevented by pre-stenting.

Objectives: To evaluate the short-term and mid-term effects of pre-stenting prior to intentional stent fracture on vessel size and integrity in a piglet model.

Methods: Five months after 14 low-profile stents (Cook Formula 418 stents) were implanted in the aorta of four piglets, they were intentionally fractured using ultra-high-pressure balloons with (pre-stent group) or without (single stent group) with another stent placed inside.

Results: Compared with the single stent group, the prestent group showed a significantly larger vessel lumen area (109 mm2 (89-141) vs. $57 \mathrm{~mm} 2(47-73), P=0.019)$, less mid-term luminal diameter loss (44\% (26-59) vs. $75 \%(62-85), P=0.007)$, lack of strut protrusion, and improved endothelialization (100\% (89-100) vs. $73 \%$ (56-96), $P=0.022$ ). Vessel wall injury was similar between groups at the time of stent fracture; however, the injury score was significantly improved at mid-term in the pre-stent group compared with the single stent group $(P=0.046)$. No damage to the external part of the blood vessels or the surrounding soft tissue was noted in either group.
\end{abstract}

Fax +1 2037853346

E-Mail: jshd@scienceinternational.org

http://structuralheartdisease.org/ (c) 2017 Journal of Structural Heart Disease Published by Science International Corp. ISSN 2326-4004

Accessible online at: http://structuralheartdisease.org/
Conclusion: Pre-stenting before intentional stent fracture may provide advantages including larger vessel diameter, maintained vessel patency, more complete endothelialization, and lack of stent strut protrusion.

Copyright (c) 2017 Science International Corp.

\section{Key Words}

Stent Fracture • Coarctation • Intentional stent fracture

\section{Introduction}

Stent implantation in younger patients is facilitated by the availability of low-profile stents that are deliverable through small delivery sheaths, although these smaller stents cannot be dilated to match an adult vessel size [1-3]. Several in vitro studies demonstrate that small- and medium-size stents can be fractured using ultra-high-pressure balloons $[4,5]$. Recently, an in vivo model of stent fracture (i.e., "unzipping") confirmed the feasibility of intentional fracture of several different stents in pigs; however, the report did not mention vessel patency after fracture and admitted to significant vessel injury secondary to intentional fracture, with Cook Formula stents associated with a slightly lower vessel injury score than other stents

* Corresponding Author:

Howaida el-Said, MD, PhD

Rady Children's Hospital

University of California San Diego

3020 Children's Way, San Diego, CA 92123, USA

Tel. +1 914409 3091; Fax: +1 858966 7903; E-Mail: hgelsaid@yahoo.com 
Table 1. Group demographics.

\begin{tabular}{|c|c|c|c|}
\hline & Re-stent group & $\begin{array}{l}\text { Single stent } \\
\text { group }\end{array}$ & $p$-value \\
\hline & $n=7$ & $n=7$ & \\
\hline Piglet weight (kg) & 6.6 & 8.1 & 0.0342 \\
\hline \multicolumn{4}{|l|}{ Original Stent } \\
\hline Type & Formula 418 & Formula 418 & \\
\hline \multicolumn{4}{|l|}{ Stent Size $(\mathrm{mm})^{*}$} \\
\hline 4 & $n=3$ & $n=1$ & \\
\hline 5,6 & $n=4$ & $n=4$ & \\
\hline 7,8 & $n=0$ & $n=2$ & \\
\hline \multicolumn{4}{|l|}{$\begin{array}{l}\text { Fracture diameter } \\
(\mathrm{mm})\end{array}$} \\
\hline 12 & $n=3$ & $n=1$ & \\
\hline 16 & $n=4$ & $n=4$ & \\
\hline 20 & $n=0$ & $n=2$ & \\
\hline $\begin{array}{l}\text { Fracture atm } \\
\text { (mean) }\end{array}$ & $14.7 \pm 4.9$ & $18.4 \pm 4.8^{* *}$ & 0.1766 \\
\hline \multicolumn{4}{|c|}{$\begin{array}{l}\text { * All Formula } 418 \text { stents were } 12 \mathrm{~mm} \text { in length } \\
\text { ** Higher atm for the single stent group can be explained by the fact that the } \\
\text { stents were thinner and harder to visualize in the older pig, so more atmo- } \\
\text { spheres were used to insure that they were broken. }\end{array}$} \\
\hline
\end{tabular}

and the EV3 stent having the lowest score [6].

Five intentional longitudinal stent fractures were reported in humans using high-pressure balloons without immediate adverse events [7]. In situ spontaneous stent fracture in humans is not uncommon and has been reported in up to $21 \%$ patients, with resultant obstruction in $80 \%$ (of which 39\% were considered severe). Some fractures can cause stent collapse, hemodynamic compromise, and embolization of stent fragments, requiring additional intervention in $75 \%$ of cases $[8,9]$. In a large report of the spontaneous fracture of 3,650 stents, there was a $42 \%$ incidence of in-stent restenosis and $4.6 \%$ incidence of thrombosis [10]. Furthermore, in a study demonstrating the feasibility of intentional stent fracture in humans, there was a significant incidence of complications (15\%), including embolization of stent fragments, unstable stent fracture, vascular tear, non-obstructive intimal tear, and aorto-pulmonary window. All complications except embolization were prevented by pre-stenting [11].
The purpose of the present study was to evaluate the impact of pre-stenting prior to intentional stent fracture in a piglet model.

\section{Methods}

\section{Study Design}

Experiments were performed using four 6-9-weekold piglets (7-11 kg), in which the aorta at various levels measured $5.7-10 \mathrm{~mm}$. A total of 14 Cook Formula 418 stents (al1 $2 \mathrm{~mm}$ length) were placed in the four piglets (three stents in two piglets and four stents in two piglets) at a diameter $1-3 \mathrm{~mm}$ larger than the vessel size to prevent stent migration. The pigs were allowed to grow for 5 months, during which time the aorta above and below the stent grew to 10.9-22 $\mathrm{mm}$, with the area where the stent was placed being locked at its original size.

In two pigs (one with four stents and the other with three stents), the previously stented area was fractured using ultra-high-pressure Atlas balloons (Bard Peripheral Vascular, Temple, AZ, USA) without pre-stenting (single stent group). The Atlas balloons were over-sized for the region of the stent but matched the size of the adjacent vessel diameter.

In the other two pigs (one with four stents and the other with three stents), the previously stented area was pre-stented prior to stent fracture using Cordis Palmaz Genesis 1910 B peripheral stents (Cordis, Hialeah, FL, USA; pre-stent group). The Genesis stents were mounted on Cordis Powerflex (Cordis, Hialeah, FL, USA) or NuMED BIB (NuMed Inc., Hopkinton, NY, USA) balloon catheters. The Genesis stents were selected for the second set of stents because they are commonly used in the pediatric population and can be ultimately dilated to reach adult vessel size. Balloon size was selected to match the adjacent vessel diameter. Balloon catheters were inflated to their respective recommended maximum pressure to place the Genesis stents inside the smaller Cook Formula stents. High-pressure Atlas balloons were then used to dilate the secondarily placed Genesis stents with simultaneous intentional fracture of the smaller Cook Formula stents.

Two pigs (short-term group; one each from the single stent and pre-stent groups, total of seven stents) were euthanized immediately and the tissue 


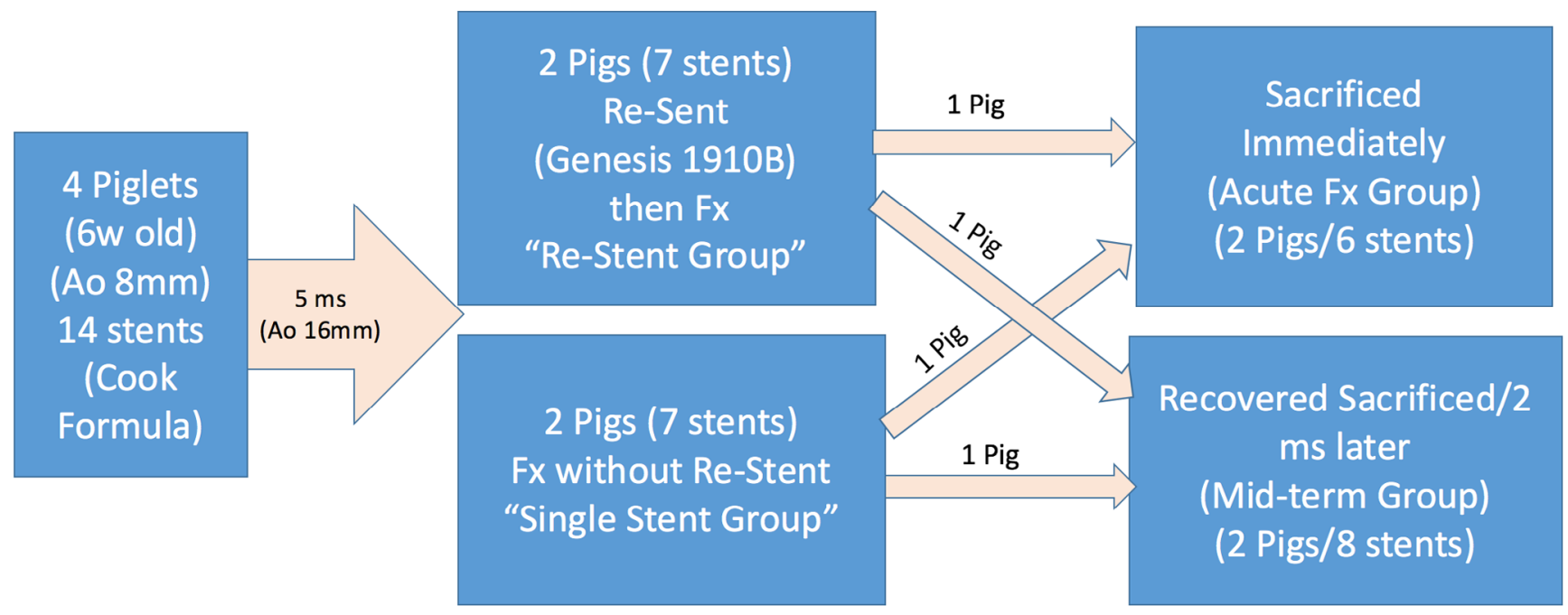

Figure 1. Flow chart showing the number of pigs and stents, group assignment, and study progression.

examined. The other two pigs (mid-term group; one each from the single stent and pre-stent groups, total of seven stents) were allowed to grow for 2 another months (Table 1, Figure 1). We chose 2 months because previous studies show complete endothelialization of injured vessels in rabbits and pigs at 28 days [12-14].

\section{Animal Experiments}

Animal experiments were approved by the Institutional Animal Care and Use Committee at Purdue University. Yorkshire Cross piglets were used to allow rapid growth over a short period of time. Survival surgeries (i.e., catheterizations) were carried out at Purdue University facilities in collaboration with Cook Research Incorporated and Cook Medical. Pigs underwent a quarantine period and were examined by a veterinarian prior to catheterization procedures and weekly throughout the duration of the study.

Pigs were started on aspirin and clopidogrel 3 days prior to catheterization. Pigs were pre-medicated with tiletamine and zolazepam, and anesthesia was induced by a mixture of ketamine and xylazine. Pigs were intubated, and anesthesia was maintained with isoflurane $(1.25-1.75 \%$ in $1.5-2.5 \mathrm{~L} / \mathrm{min}$ oxygen) via a standard rebreathing anesthetic circuit for the remainder of the procedures. Intravascular access was obtained by carotid or femoral cut-down or percutaneous puncture using a modified Seldinger technique. Intramuscular antibiotic (ceftiofur crystalline) was given prior to stent implantation. After the procedure, anesthesia was discontinued, and pigs recovered on a raised floor pen. Pigs were medicated as necessary (meloxicam, butorphanol, or flunixin) to assure uncomplicated recovery and monitored every 15-30 min until they were alert and responsive. After catheterization, pigs were maintained on aspirin and clopidogrel throughout the growth phase to prevent stent thrombosis. Clinically, pigs were healthy and fully ambulatory throughout the study. Pig weight ranged from $9-11 \mathrm{~kg}$ at initial stent implantation to 98-119 kg at the time of euthanasia.

For euthanasia, pigs were anesthetized as above. Sodium nitroprusside was administered to reduce post-mortem vasospasm, and isoflurane level was increased to $5 \%$. After approximately $5 \mathrm{~min}$, pigs were euthanized by intravenous administration of potassium chloride. Death was verified by a lack of vital signs. After angiography and euthanasia, the abdominal cavity was opened and the aorta exposed. After gross examination of the aorta, the left ventricle and venous systems were cannulated. The aorta was perfusion-flushed with physiological saline until the effluent began to run clear. The stented area was then perfusion-fixed with Prefer fixative for approximately 10 min prior to immersion fixation in $10 \%$ neutral buffered formalin.

After the aorta was removed, pigs were submitted for full postmortem evaluation. Detailed macroscopic examination was performed by a board-certified vet- 

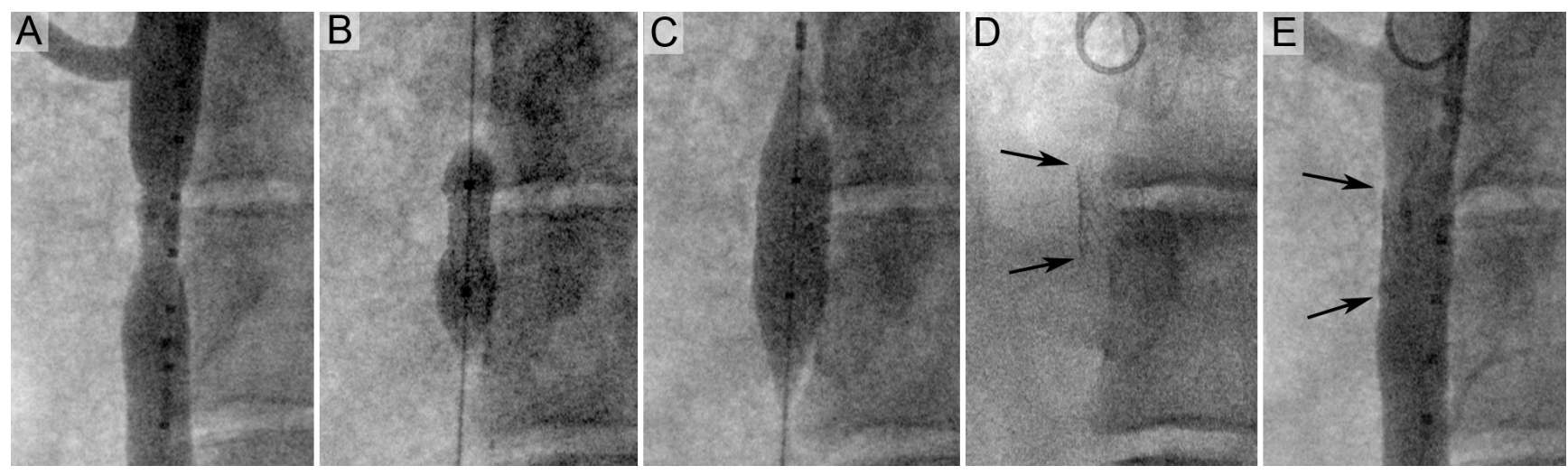

Figure 2. Pre-stenting technique. Panel A. Angiogram of the abdominal aorta with a 4-mm Cook Formula 418 stent implanted 5 months prior (stent diameter: $7.3 \mathrm{~mm}$ ). Panel B. Implantation and anchoring of a Palmaz Genesis 1910 B stent mounted on a 10$\mathrm{mm}$ Powerflex balloon. Panel C. Intentional fracture of the previously implanted Cook stent with simultaneous dilation of the newly implanted Palmaz Genesis stent using an ultra-high-pressure 12-mm Atlas balloon. Panel D. Radiograph showing the integrity and position of the newly implanted Palmaz Genesis stent inside the previously implanted Cook stent. Panel E. Angiogram showing vessel patency and minimal in-stent restenosis at the edges of the Palmaz Genesis stent 2 months after implantation (stent diameter: 9.9 $\mathrm{mm}$ ). Black arrows indicate the edges of the Palmaz Genesis stent.

erinary pathologist, who assessed the stented blood vessel and adjacent areas for evidence of macroscopic tissue damage such as bleeding, contusion, rupture, and scarring. Major body systems including the brain, thoracic cavity viscera, peritoneal viscera, and hind limb muscles were examined grossly. Hind limb muscles were sectioned serially. In addition to stented regions of the aorta, a standard list of 33 tissues, as well as any grossly visible or suspected lesions, were collected for histopathology. Five muscle groups from each hind limb, lymph nodes regional to the hind limbs and descending aorta, and multiple sections of brain, lung, each kidney, liver, heart, spleen, and spinal cord were microscopically examined for any embolic lesion or stent fragment displacement.

\section{Intentional Stent Fracture and Pre-Stenting Techniques}

Serial dilation of small-diameter pre-mounted stents with intentional stent fracture and pre-stenting was feasible in a growing piglet model that mimics physiologic changes occurring in the vasculature of growing children. Small-diameter pre-mounted stents were successfully implanted in small vessels (Figure 2A). As expected, the patency of the growing blood vessel at the level of the stent was reduced by the previously implanted stents (Figure 2A); however, the vessel above and below the stent grew to almost double the original size of the vessel that held the original stent. Low-pressure balloons did not fully dilate and fracture the stents (Figure 2B), but ultra-high-pressure balloons achieved intentional stent fracture and re-dilation of previously implanted stents (Figure 2C). Stent dilation was performed in increments, and fracture occurred at the previously noted fracture diameter determined by bench testing [4]. In the single stent group, the stents were thinner and more difficult to visualize in the older pigs, so more atmospheres were used to ensure their breakage.

\section{Radiographic, Angiographic, Gross Inspection, X-ray,} Computed Tomographic, and Histopathologic Evaluation

Radiographic images and angiograms were obtained during all catheterization procedures. After euthanasia, necropsy was performed for gross inspection, histologic, and radiographic evaluation. The stented vessels were flushed with physiological saline for approximately $10 \mathrm{~min}$ followed by perfusion of Prefer fixative for $15 \mathrm{~min}$ prior to excision. Vessel diameter was measured using angiograms, and vessel luminal area was assessed using histologic samples.

Radiography. High-resolution radiography and micro computed tomography (CT) were performed on all stents after explant. High-resolution radiography was performed using a Kubtec Xpert 80-L w/ 


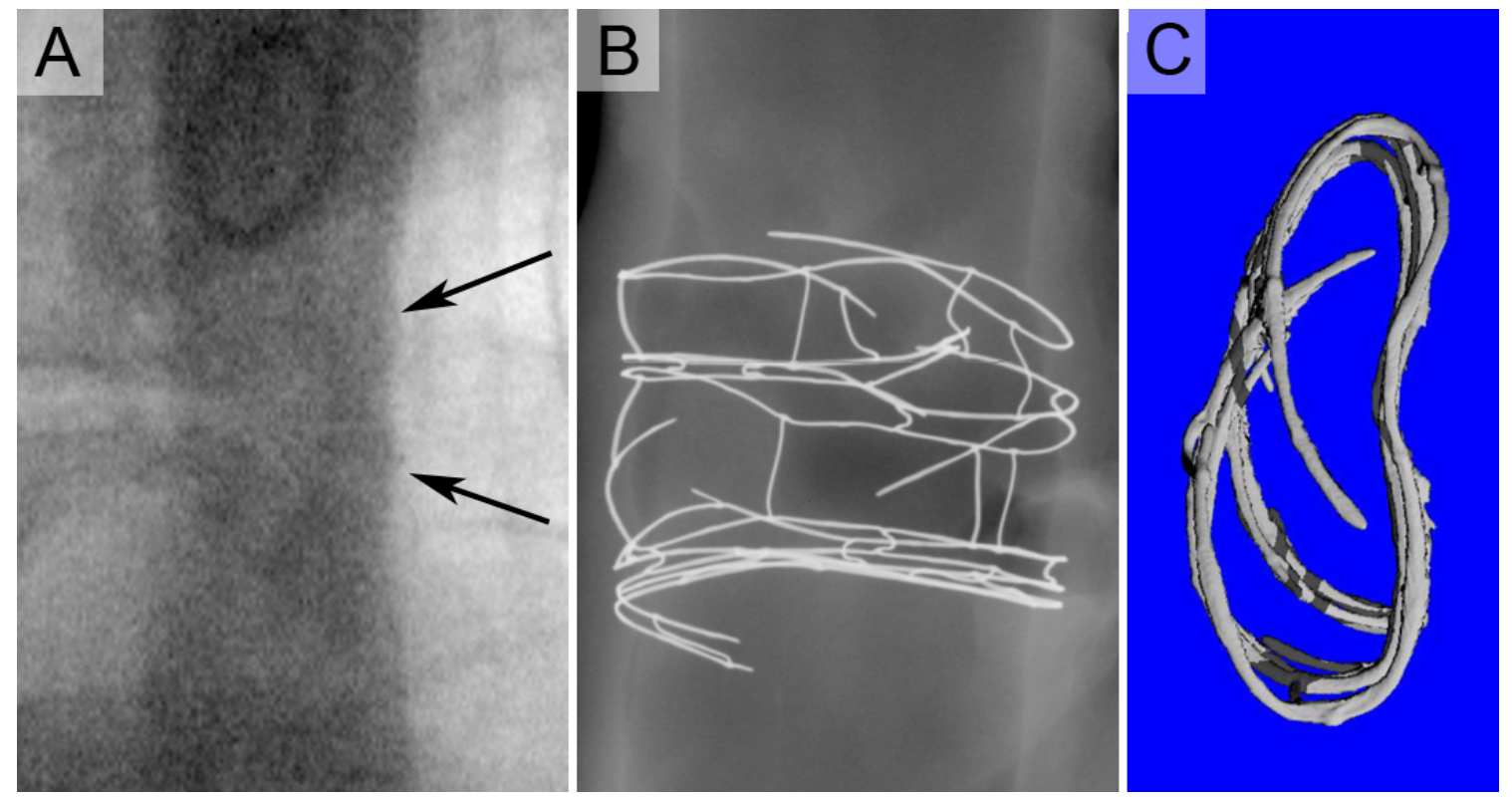

Figure 3. Reduced vessel patency and stent integrity after intentional fracture without pre-stenting. Panel A. Angiogram showing a small decrease in the diameter of the stented vessel segment compared with the adjacent area 2 months after intentional fracture and re-dilation of a 6-mm Cook Formula 418 stent with an ultra-high-pressure 16-mm Atlas balloon. Black arrows indicate the edges of the Cook stent. Panel B. High-resolution radiograph of the same segment showing fractured circumferential struts. Panel $C$. Downthe-barrel view of a three-dimensional rendition CT image of the same stent showing severely compromised luminal diameter in the antero-posterior dimension with struts protruding into the vessel lumen.

DIGI-VIEW 400, and micro CT was performed using a Scanco VivaCT40 System.

Histopathology. Perfusion-fixed, excised arteries were further immersion-fixed in $10 \%$ neutral buffered formalin. After dehydration though a serial gradient of alcohols and clearing in xylene, tissues were infiltrated with methyl methacrylate and polymerized in a water bath. Plastic embedded arteries, with stents in place, were cut on a diamond-encrusted saw blade into 30-50 $\mu \mathrm{m}$ sections. Proximal distal sections of stented artery (taken at $25 \%$ and $75 \%$ of the stent length) were stained with hematoxylin and eosin using routine techniques. Proximal and distal reference sections were trimmed from the fixed arteries, processed using routine paraffin histology techniques, and stained with hematoxylin and eosin. Morphometric measurements of each cross-section included vessel lumen area. Histopathological evaluation of stented vessels was performed by a board-certified veterinary pathologist. Cross-sections within each stent were evaluated for injury using an established semi-quantitative scoring system based on industry-standard consensus recommendations [15]. At each strut within a cross-section, vessel wall injury was scored as follows: 0 for no change in internal elastic lamina, 1 for rupture of internal elastic lamina, 2 for injury to tunica media, or 3 for injury to external elastic lamina and extending into or through the tunica adventitia. Each vessel had two cross-sections. The score for each cross-section was the sum of all injury scores for that cross-section divided by the number of total struts in the cross-section. The injury score for each group is the injury score for all cross-sections for the group divided by the total number of cross-sections for the group. Endothelialization was determined by the histological presence of endothelium over each strut within a cross-section. The degree of endothelialization is the percentage of endothelialized struts in the vessel.

\section{Statistical Analysis}

Descriptive statistical analysis was used to compare variables between pre-stent and single stent groups; data are expressed as median and interquartile range. Mann-Whitney tests were used to compare vessel lumen area, balloon size, degree of endothelialization, 

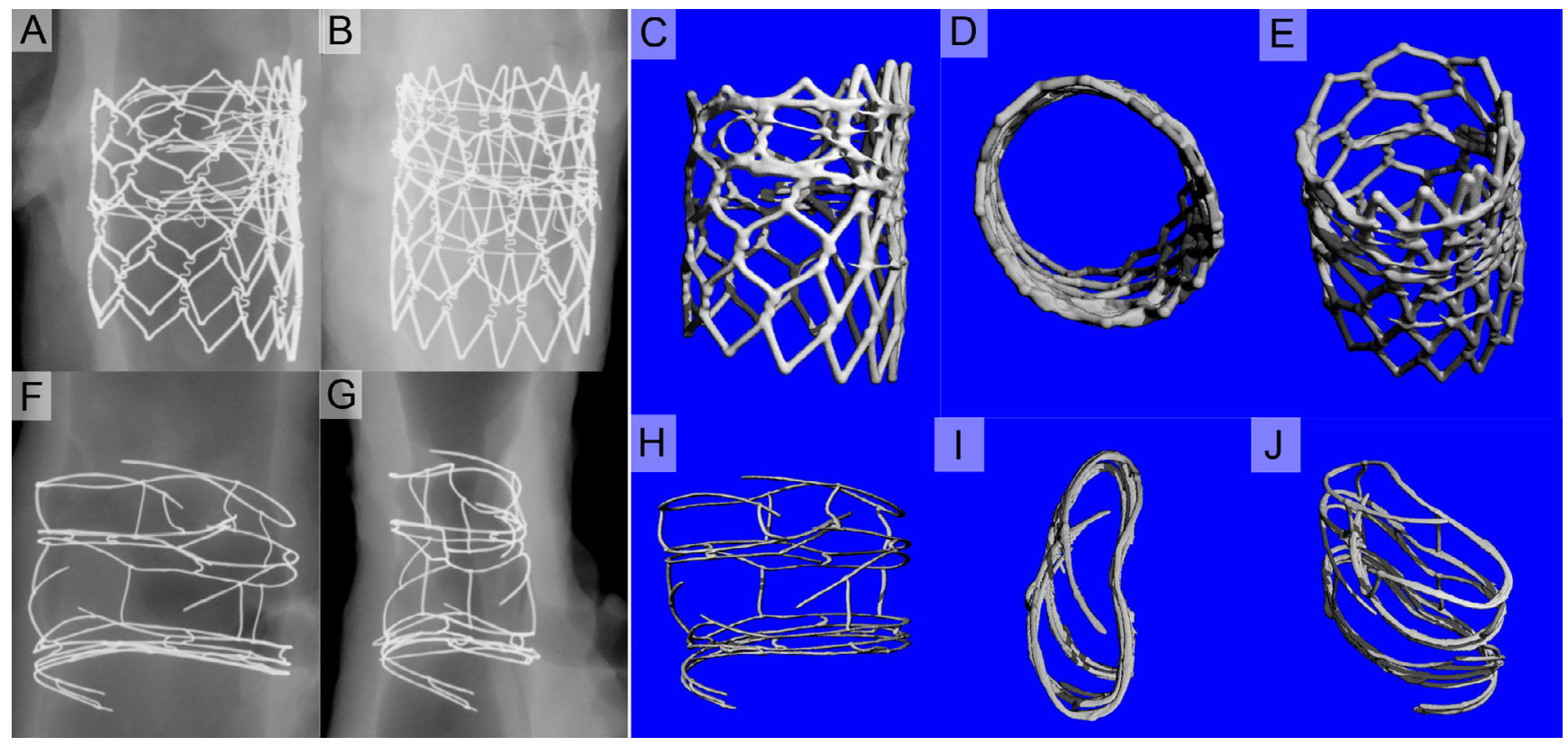

Figure 4. High-resolution radiographic and three-dimensional rendition CT images comparing pre-stenting at the time of stent fracture and single stent fracture. Intact stent integrity in the pre-stented segment maintained vessel patency, whereas loss of stent integrity after single stent fracture caused in-folding and compromised vessel lumen patency. Panels A, B, C, D, and E. Palmaz Genesis 1910 B stent implanted inside a Cook Formula 418 stent with simultaneous intentional fracture of the Cook stent. Images were taken 2 months after intentional fracture of the Cook stent. Images show intact internal stent integrity of the pre-stented vessel segment 2 months after implantation of the Palmaz Genesis stent with simultaneous intentional fracture of the 5-month previously implanted Cook stent. Panels F, G, H, l, and J. Cook Formula 418 stent 2 months after stent fracture (i.e., unzipping) without pre-stenting. Images show compromised stent integrity and vessel patency of the stented segment 2 months after intentional stent fracture and re-dilation of the 5-month previously implanted Cook stent.

Table 2. Comparison of outcome measures between the re-stent and single stent groups.

\begin{tabular}{llll}
\hline & Re-stent group & $\begin{array}{l}\text { Single stent } \\
\text { group }\end{array}$ & $p$-value \\
\hline $\begin{array}{llll}\text { Lumen area } \\
\left(\mathrm{mm}^{2}\right)^{*} \text { of stented } \\
\text { blood vessels }\end{array}$ & $\begin{array}{l}108.9(88.7- \\
(\mathrm{n}=4)\end{array}$ & $\begin{array}{l}56.8(47.3- \\
72.8)\end{array}$ & 0.0190 \\
$\begin{array}{l}\text { Vessel diameter } \\
\text { loss (\%) }\end{array}$ & $\begin{array}{l}44(26-59) \\
(\mathrm{n}=4)\end{array}$ & $\begin{array}{l}75(61-85) \\
(\mathrm{n}=4)\end{array}$ & 0.0065 \\
$\begin{array}{l}\text { 2 months after } \\
\text { dilation }\end{array}$ & & & \\
$\begin{array}{l}\text { Endothelialization } \\
\text { (\%) of stent struts }\end{array}$ & $\begin{array}{l}100(89-100) \\
(\mathrm{n}=8)\end{array}$ & $\begin{array}{l}73(56-96) \\
(\mathrm{n}=8)\end{array}$ & 0.0221 \\
$\begin{array}{l}\text { Vessel wall injury } \\
\text { score per struts }\end{array}$ & $\begin{array}{l}0.3(0.26-0.45) \\
(\mathrm{n}=8)\end{array}$ & $\begin{array}{l}0.5(0.35-0.63) \\
(\mathrm{n}=8)\end{array}$ & 0.0673 \\
& & & \\
\hline
\end{tabular}

Values expressed as median and interquartile range.

* Only the stents dilated with $16 \mathrm{~mm}$ balloon for diameter conformity

and vessel wall injury scores between groups; data are expressed as median and interquartile range. Mann-Whitney and Wilcoxon rank tests were used to compare the loss of luminal diameter from implantation to mid-term between groups; data are expressed as median change (\%) and interquartile range. Vessel wall injury scores were compared between groups using unpaired t-tests and Mann-Whitney post-hoc tests for nonparametric data. Analyses were performed using Excel (Microsoft, Redmond, WA), GraphPad Prism (GraphPad, La Jolla, CA), and Instat 3 (Graph Pad, San Diego, CA) software. $P$-values $<0.05$ were considered statistically significant.

\section{Results}

Vessel Patency and Luminal Diameter after Intentional Stent Fracture

After intentional stent fracture, we found significant vessel diameter loss in the single stent group 
A

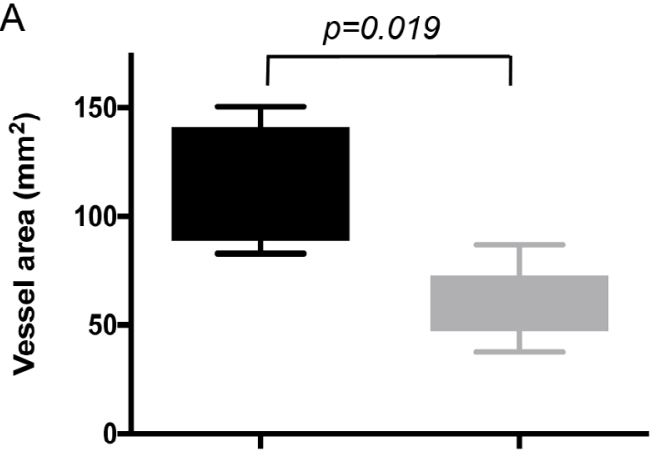

Pre-stent group Single stent group

C

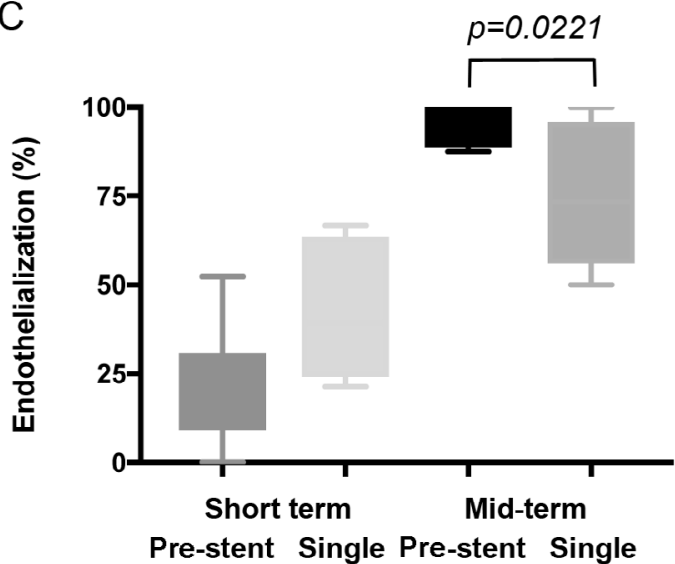

B

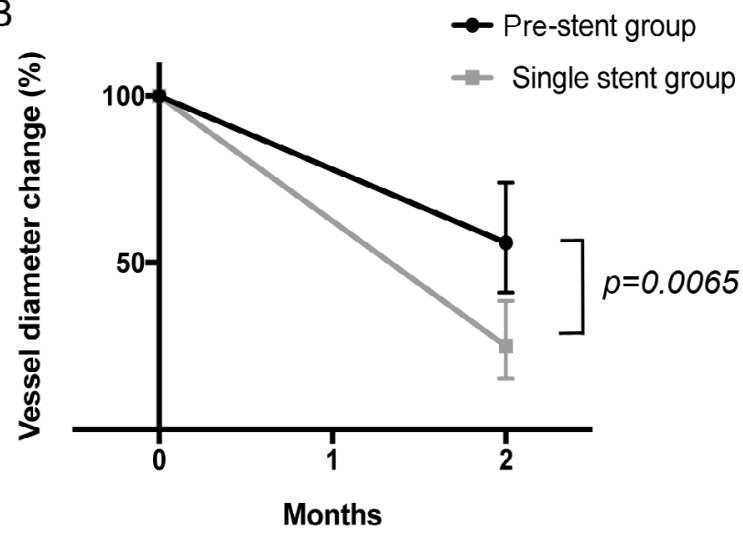

D

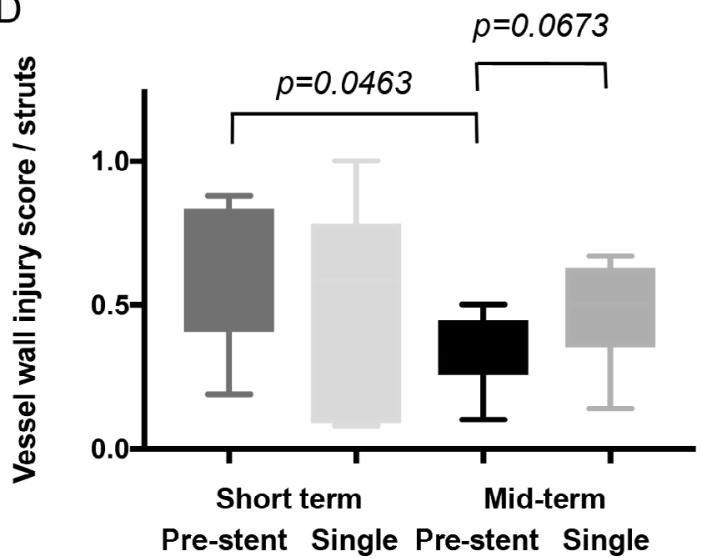

Figure 5. Comparison of vessel lumen diameter change, endothelialization, and vessel wall injury after intentional stent fracture between pre-stent and single stent groups. Panel A. Histomorphometric comparison of stented vessel lumen area 2 months after intentional stent fracture. The area of the stented vessel segments was significantly larger in the pre-stent group than in the single stent group. Panel B. The pre-stent group maintained larger angiographic lumen diameter, whereas the single stent group exhibited significant diameter loss due to stent in-folding and collapse 2 months after balloon angioplasty. Panel C. Pre-stenting allowed almost complete endothelialization 2 months after stent implantation, whereas endothelial coverage was significantly less in the single stent group due to luminal stent strut protrusion. Panel D. Comparison of vessel injury between pre-stent and single stent groups at short-term and mid-term evaluation. Vessel wall injury was similar at the time of stent fracture regardless of pre-stenting or unzipping without pre-stenting; however, injury score significantly improved 2 months after stent fracture in the pre-stent group but not in the single stent group. Data are shown as median, interquartile range, and range.

compared with the pre-stent group (Table 2). The degree of vessel diameter loss was subtle on traditional angiography (Figure 3A), but high-resolution radiography and rotational $C T$ showed compromised vessel patency (Figure 3B and 33C) and revealed the mechanisms of vessel diameter loss in the single stent group as including stent in-folding, buckling, and collapse. This stent in-folding, buckling, and collapse was not present in the pre-stent group (Figure 4).

Consistent with the maintained vessel diameter as shown by angiography, radiography, and CT, the lu- minal area of the stented vessel segments measured by histomorphometry was significantly larger in the pre-stent group (109 $\left.\mathrm{mm}^{2}(89-141)\right)$ than in the single stent group (57 $\mathrm{mm}^{2}$ (47-73), $P=0.019$; Figure $5 \mathrm{~A}$ ). The angiographic vessel diameter of stented vessel segments was similar to that of adjacent naïve vessel segments in the pre-stent group, whereas the single stent group demonstrated significantly larger luminal diameter loss. Two months after angioplasty, compared with the balloon diameter used for dilation (we only compared segments after dilation with 16 
$\mathrm{mm}$ for consistency of measurements), the pre-stent group showed only 44\% (26-59) angiographic luminal diameter loss, whereas the single stent group showed 75\% (62-85) luminal diameter loss $(P=0.007$; Figure 5B).

\section{Endothelialization and Exposed Stent Struts}

There were no stent struts protruding into the lumen of vessels in the pre-stent group (Figure 4 and 6), whereas numerous naked (i.e., non-endothelialized) stent struts were noted in the lumen of vessels in the single stent group (Figures 6G, 6H, and 6I). Accordingly, the degree of endothelialization was markedly higher in the pre-stent group (100\% (89-100)) than in the single stent group (73\% (56-96), $P=0.022$; Table 2, Figure 5C).

\section{Vessel Wall Injury}

Similar degrees of vessel wall injury were noted in the pre-stent group and single stent group at the time of intentional fracture (i.e., short-term) with scores of 0.51 and 0.59 , respectively. However, at mid-term evaluation, the injury score in the pre-stent group was significantly improved (decrease from 0.51 to $0.31, P=0.046$ ), whereas the injury score in the single stent group was unchanged $(0.5, P>0.05$; Figure 5D), although the difference between groups did not reach statistical significance $(P=0.067$; Table 2, Figure 5D).

\section{Lack of Injury to Adjacent Soft Tissue}

Macroscopic examination of stented areas revealed a lack of external stent strut protrusion or transmural vessel injury in both the pre-stent and single stent groups (Figure 6). In both groups, the broken stent struts remained embedded in the vessel wall or exhibited inward protrusion due to the in-folding collapse of fractured stents (Figure 3, 4, and 6). There was no damage to the external part of the blood vessels or surrounding soft tissue (Figure 6C).

Microscopic examination of five muscle groups in the hind limb, lymph nodes regional to the hind limbs and descending aorta, and multiple sections of brain, lung, each kidney, liver, heart, spleen, and spinal cord showed no important lesions and no evidence of thromboembolism or stent fragment displacement in any downstream (i.e., hind limb muscles/regional lymph nodes) or systemic tissues.

\section{Discussion}

Our study demonstrates the feasibility of intentional stent fracture of previously implanted stents with simultaneous pre-stenting in an in vivo piglet model. Additionally, pre-stenting appears to provide appropriate vessel patency, prevents vessel diameter loss, improves endothelialization rate, and prevents intraluminal protrusion of stent struts. Moreover, pre-stenting appears to be safe, with no damage to surrounding soft tissue and no significant vessel wall injury.

Stented vessel diameter and cross-sectional area were significantly improved by pre-stenting during simultaneous intentional stent fracture of previously placed stents compared with no pre-stenting. Intentional stent fracture causes a loss of stent integrity with a dramatic decline in radial stiffness and strength [4]. This decrease in radial stiffness and strength allows the blood vessel to recoil more than with an intact stent. Moreover, an irregular stent fracture pattern may result in an irregular vessel wall shape. Both recoil and irregular shapes of blood vessels lead to compromised vessel lumen with decreased vessel diameter and cross-sectional area, consistent with our findings. As stent implantation is aimed at recovering areas of stenoses, it is of utmost importance that the stented vessel maintains appropriate patency. Simple stent fracture (i.e., unzipping) without the additional benefit of pre-stenting may not stabilize the vessel wall and may decrease vessel patency, thereby leading to restenosis and a significant pressure gradient.

Endothelial coverage allows appropriate blood vessel function and prevents thrombus formation at the vessel wall [16]. Fractured and not pre-stented fragments may protrude into the vessel lumen and prolong or even prevent complete endothelialization. Pre-stenting at the time of intentional fracture may prevent stent strut protrusion and thereby improve the chance of complete endothelialization with more rapid re-institution of physiologic vascular endothelial function.

Our observed vessel wall injury during pre-stenting was comparable to that after balloon angioplasty or single stent placement [6]. Incidental fracture of stents may cause severe vessel wall injury [17], and even intentional stent fracture in vivo (i.e., unzipping) may cause vessel wall damage due to an irregular 

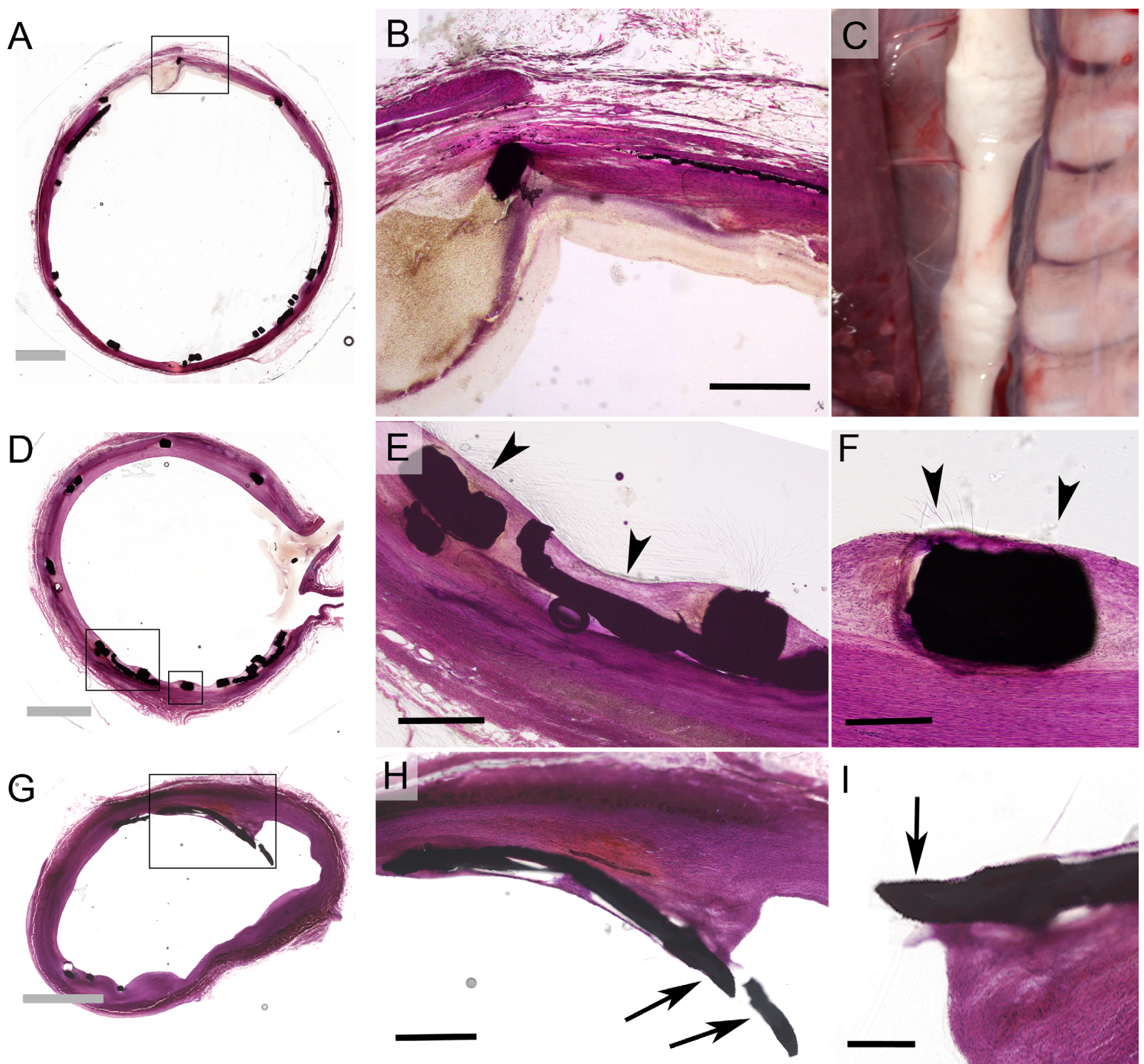

Figure 6. Vessel wall injury and endothelialization of blood vessels after pre-stenting compared with single stent implantation. Panels A, B, and C. Intentional stent fracture with pre-stenting caused only minimal vessel wall injury and did not result in transmural vessel injury or damage of surrounding soft tissue. Panel A. Localized dissection of the tunica media in a stented vessel immediately after stent implantation and lack of external protrusion of stent struts. Panel B. High-magnification image showing the extent of dissection and intramural hemorrhage immediately after stent placement. Panel C. Macroscopic picture showing no damage to the external surface of blood vessels despite the size discrepancy of stented segments. Panels D, E, and F. Pre-stenting allowed appropriate endothelialization and minimal vessel wall injury 2 months after stent placement. Panel D. Mid-term (i.e., 2 month) follow-up showed mild intimal and medial thickening in response to stent placement, no intraluminal protrusion of stent struts, and no dissection or pathologic vessel dilation. Panels E and F. High-magnification images showing almost complete endothelial coverage over stent struts 2 months after implantation. Panel G. Single stent implantation with intentional stent fracture (i.e., unzipping without pre-stenting) led to incomplete endothelialization due to intraluminal stent strut protrusion and decreased vessel lumen cross-sectional area. Panels H and I. High-magnification images showing stent strut protrusion without endothelial coverage. Grey bars in A, D, and G $=3$ $\mathrm{mm}$; black bars in $\mathrm{B}, \mathrm{E}$, and $\mathrm{H}=0.5 \mathrm{~mm}$ and in $\mathrm{F}$ and $\mathrm{I}=0.2 \mathrm{~mm}$. Black arrowheads indicate endothelial coverage over stent struts, and black arrows point to bare metal struts protruding into the vessel lumen. 
fracture pattern [11]. In our experience, pre-stenting at the time of intentional stent fracture caused no further injury to the vessel wall, and injury scores improved at mid-term evaluation.

Macroscopic examination of stented vessels provided no evidence of damage to the surrounding tissue or transmural vessel injury. This is important because in vitro testing of stents with intentional fracture produces an irregular fracture pattern, with many stent struts protruding tangentially [13]. These externally protruding stent fragments can penetrate through blood vessels and damage adjacent soft tissue, including arteriovenous malformations [11, 18, 19]. However, pre-stenting allows realignment of the struts of intentionally broken stents, which remain embedded in the vessel wall, in a more ideal circular fashion.

Our study has some limitations. The experiments were performed in a growing piglet model that has different growth velocity characteristics than humans. Nevertheless, this model has been employed in similar studies to mimic the growth of infants and small children [6]. Although the number of stents and animals were small, they were adequate to achieve statistical significance in the areas demonstrated. We assessed only one type of stent in this in vivo study, which was selected based on its superior characteristics in our in vitro bench testing [4]. Pressure gradients across the stents were not measured in all animals because we found only very small $(2-5 \mathrm{~mm}$ $\mathrm{Hg}$ ) gradients across stented segments in the few we measured. Moreover, our model was not intended to create significant coarctation with pressure gradients; rather, it was designed to evaluate the feasibility and characteristics of the pre-stenting technique.

In conclusion, our study shows that pre-stenting at the time of intentional pre-existing stent fracture provides advantages over simple stent fracture. Pre-stented vessels had larger vessel diameters, maintained better vessel patency, had more complete endothelialization, and showed no protrusion of stent struts into vessel lumens. These findings should be considered in small children requiring vessel angioplasty or stenting.

\section{Acknowledgements}

We deeply appreciate and thank the Foroni Family of Madrid, Spain for their generous contributions supporting this work and related stent studies. We thank William Schoenlein, Melissa Bible, Tracy Moller, Gena Brock, and Richard Sieber for their contributions and assistance with animal experiments. We also thank Omar El-Sabrout for his input in the study and review of the manuscript.

\section{Conflict of Interest}

The authors have no conflict of interest relevant to this publication.

\section{Comment on this Article or Ask a Question}

\section{References}

1. Mullins $C E$, $\mathrm{O}^{\prime}$ Laughlin MP, Vick GW 3rd, Mayer DC, Myers TJ, Kearney DL, et al. Implantation of balloon-expandable intravascular grafts by catheterization in pulmonary arteries and systemic veins. Circulation. 1988;77:188-199. PMID: 3335067

2. Rosenthal E, Qureshi SA. Stent implantation in congenital heart disease. Br Heart J. 1992;67:211-212. PMID: 1554537

3. O'Laughlin MP, Slack MC, Grifka RG, Perry SB, Lock JE, Mullins CE. Implantation and intermediate-term follow-up of stents in congenital heart disease. Circulation. 1993;88:605-614. PMID: 8339424

4. Bratincsak A, Moore JW, Gulker B, Choules B, Koren L, El-Said HG. Breaking the limit:
Mechanical characterization of overexpanded balloon expandable stents used in congenital heart disease. Congenit Heart Dis. 2015;10:51-63. DOI: 10.1111/ chd. 12175

5. Sathanandam SK, Haddad LM, Subramanian S, Wright D, Philip R, Waller BR. Unzipping of small diameter stents: An in vitro study. Catheter Cardiovasc Interv. 2015;85:249-258. DOI: 10.1002/ccd.25596

6. Sathanandam SK, Kumar TK, Hoskoppal D, Haddad LM, Subramanian S, Sullivan RD, et al. Feasibility and safety of unzipping small diameter stents in the blood vessels of piglets. JACC Cardiovasc Interv. 2016;9:11381149. DOI: $10.1016 /$ j.jcin.2016.02.035
7. Patel $M$, Justino $H$. Intentional stent fractures in structural heart disease: When breaking the chains is the only way! Catheter Cardiovasc Interv. 2013;81:179. DOI: $10.1002 / \mathrm{ccd} .24762$

8. McElhinney DB, Bergersen L, Marshall AC. In situ fracture of stents implanted for relief of pulmonary arterial stenosis in patients with congenitally malformed hearts. Cardiol Young. 2008;18:405-414. DOI: 10.1017/S1047951108002424

9. Breinholt JP, Nugent AW, Law MA, Justino $H$, Mullins $C E$, Ing FF. Stent fractures in congenital heart disease. Catheter Cardiovasc Interv. 2008;72:977-982. DOI: 10.1002/ ccd. 21742 
10. Kan J, Ge Z, Zhang JJ, Liu ZZ, Tian NL, Ye F, et al. Incidence and clinical outcomes of stent fractures on the basis of 6,555 patients and 16,482 drug-eluting stents from 4 centers. JACC Cardiovasc Interv. 2016;9:1115-1123. DOI: 10.1016/j.jcin.2016.02.025

11. Morray BH, McElhinney DB, Marshall $A C$, Porras D. Intentional fracture of maximally dilated balloon-expandable pulmonary artery stents using ultra-high-pressure balloon angioplasty: A preliminary analysis. Circ Cardiovasc Interv. 2016;9:e003281. DOI: 10.1161/CIRCINTERVENTIONS.115.003281

12. Van Belle E, Tio FO, Couffinhal T, Maillard L, Passeri J, Isner JM. Stent endothelialization. Time course, impact of local catheter delivery, feasibility of recombinant protein administration, and response to cytokine expedition. Circulation. 1997;95:438-448. PMID: 9008462

13. Taylor AJ, Gorman PD, Kenwood B, Hudak C, Tashko G, Virmani R. A comparison of four stent designs on arterial injury, cellular proliferation, neointima formation, and arterial dimensions in an experimental porcine model. Catheter Cardiovasc Interv. 2001;53:420-425. DOI: 10.1002/ccd.1194

14. Schwartz RS, Huber KC, Murphy JG, Edwards WD, Camrud AR, Vlietstra RE, et al. Restenosis and the proportional neointimal response to coronary artery injury: results in a porcine model. J Am Coll Cardiol. 1992;19:267-274. PMID: 1732351

15. Schwartz RS, Edelman E, Virmani R, Carter A, Granada JF, Kaluza GL, et al. Drug-eluting stents in preclinical studies: updated consensus recommendations for preclinical evaluation. Circ Cardiovasc Interv. 2008;1:143-153. DOI: 10.1161/CIRCINTERVENTIONS.108.789974

16. van Hinsbergh VW. Endothelium--role in regulation of coagulation and inflammation. Semin Immunopathol. 2012;34:93106. DOI: $10.1007 / \mathrm{s} 00281-011-0285-5$

17. McElhinney DB, Marshall AC, Schievano S. Fracture of cardiovascular stents in patients with congenital heart disease: theoretical and empirical considerations. Circ Cardiovasc Interv. 2013;6:575-585. DOI:
10.1161/CIRCINTERVENTIONS.113.000148

18. Bernier PL, Hallbergson A, Schachtner SK, Rome JJ, Gaynor JW. Aortopulmonary fistula after outflow tract stent in repaired truncus. Ann Thorac Surg. 2014;98:e55-e57. DOI: 10.1016/j.athoracsur.2014.06.090

19. Page $M$, Nastase $O$, Maes F, Kefer J, Sluysmans $T$, Poncelet $A$, et al. Aortopulmonary fistula after multiple pulmonary artery stenting and dilatation for postarterial switch supravalvular stenosis. Case Rep Cardiol. 2015;2015:371925. DOI: $10.1155 / 2015 / 371925$

Cite this article as: Bratincsák $A$, Van Alstine W, Koren L, Stoughton K, Negrón-Garcia J, Ragheb A, El-Sabrout $\mathrm{H}$, Moore JW, el-Said H. Intentional Fracture of Previously Placed Stents: Impact of Pre-stenting in a Piglet Model. Structural Heart Disease. 2017;3(6):165175. DOI: https://doi.org/10.12945/j. jshd.2017.030.17 